\title{
Dark energy and the hierarchy problem
}

\author{
Pisin Chen ${ }^{\text {** }}$ \\ ${ }^{a}$ Kavli Institute for Particle Astrophysics and Cosmology, \\ Stanford Linear Accelerator Center, Stanford University, Stanford CA 94309, U.S.A.
}

The well-known hierarchy between the Planck scale $\left(\sim 10^{19} \mathrm{GeV}\right)$ and the TeV scale, namely a ratio of $\sim 10^{16}$ between the two, is coincidentally repeated in a inverted order between the TeV scale and the dark energy scale at $\sim 10^{-3} \mathrm{eV}$ implied by the observations. We argue that this is not a numerical coincidence. The same brane-world setups to address the first hierarchy problem may also in principle address this second hierarchy issue. Specifically, we consider supersymmetry in the bulk and its breaking on the brane and resort to the Casimir energy induced by the bulk graviton-gravitino mass-shift on the brane as the dark energy. For the ADD model we found that our notion is sensible only if the number of extra dimension $n=2$. We extend our study to the Randall-Sundrum model. Invoking the chirality-flip on the boundaries for SUSY-breaking, the zero-mode gravitino contribution to the Casimir energy does give rise to the double hierarchy. Unfortunately since the higher Kaluza-Klein modes acquire relative mass-shifts at the $\mathrm{TeV}$ level, the zero-mode contribution to Casimir energy is overshadowed.

\section{INTRODUCTION}

The combination of recent data from WMAP (WMAP3) [1] and Sloan Digital Sky Survey (SDSS) [2] determines $w=-0.94 \pm 0.09$ for the dark energy equation of state, $p=w \rho$, while the Supernova Legacy Survey (SNLS) 3 , gives $w=-1.023 \pm 0.090$ (stat) \pm 0.054 (sys). These results do not seem to leave much freedom for ideas other than a bone fide cosmological constant $(w=-1)$. If dark energy is indeed a cosmological constant which never changes in space and time, then it must be a fundamental property of the spacetime. Observations imply that such a cosmological constant would correspond to a vacuum energy density $\rho_{\mathrm{DE}} \sim\left(10^{-3} \mathrm{eV}\right)^{4}$. One cannot but note that the energy scale of this fundamental property of the vacuum is so much smaller than that of the standard model of particle physics, which is $\sim \mathrm{TeV}$, by a factor $\sim 10^{-15}$. Why is this energy gap so huge?

There has been another long-standing hierarchy problem in physics, i.e., the existence of a huge gap between the Planck scale of quantum gravity at $10^{19} \mathrm{GeV}$ and that of the stan-

\footnotetext{
*This work is supported by the US Department of Energy under Contract No. DE-AC03-76SF00515.
}

dard model gauge interactions at $\mathrm{TeV}$, by a factor $\sim 10^{16}$. As is well-known, there have been two interesting solutions to this hierarchy problem proposed in recent years: the ArkaniHamed-Dimopoulos-Dvali (ADD) model 4 and the Randall-Sundrum (RS) model[5]. In both models the brane-world scenario is invoked where the 3-brane is imbedded in the extra dimensions, and the SM fields are confined to the brane while gravity fields reside in the bulk. In the case of ADD, the extra spatial dimensions are flat. The gravity is weak (or the Planck scale is huge) because it is diluted by the largeness of the extra dimensions in which it resides. In the alternative solution proposed by RS, the gravity is weak on the $\mathrm{TeV}$ brane because its strength is exponentially suppressed by the warp factor descended from the Planck brane.

Are these two hierarchy problems related? To address that, we notice the remarkable numerical coincidence between the two, i.e.,

$\frac{\rho_{\mathrm{DE}}^{1 / 4}}{M_{\mathrm{SM}}} \simeq \frac{M_{\mathrm{SM}}}{M_{\mathrm{Pl}}}$.

That is, the dark energy scale happens to be inverted from the Planck scale through the intermediary of the Standard Model (SM) scale, pre- 
sumably $\sim \mathrm{TeV}$. We suspect that this is not a pure coincidence. Rather, there may actually be a deeper underlying connection that results in such a manifestation. This may not be too much of a stretch. After all, the cosmological constant as a manifestation of vacuum energy is necessarily connected with the structure of spacetime and quantum fields. But Eq.(1) actually implies more. It suggests that their possible connection must be mediated and inverted by the $\mathrm{TeV}$ physics. In this paper we will rely on Eq.(1) as our guidance to explore the nature of the dark energy. We should like to caution, however, that the dark energy scale is actually not on the same footing as the other two energy scales. Whereas TeV scale represents the interaction strengths of the Standard Model and possibly its supersymmetric extension, and the Planck scale that for the gravitational interaction, the dark energy scale is not associated with any new interaction strength per se. After all, there are only four fundamental interactions in this world. It is therefore clear that the dark energy scale must not be a primary fundamental scale in physics, but rather a deduced, secondary quantity. In this regard, Eq.(1) serves to explicate the relationship of the dark energy scale with that of the four fundamental interactions. That is, we posit that the underlying mechanism that induces the dark energy must be resulted from a double suppression by the same hierarchy factor descended from the Planck scale:

$\rho_{\mathrm{DE}}^{1 / 4} \simeq \frac{M_{\mathrm{SM}}}{M_{\mathrm{pl}}} M_{\mathrm{SM}}=\left(\frac{M_{\mathrm{SM}}}{M_{\mathrm{Pl}}}\right)^{2} M_{\mathrm{Pl}}$.

In our previous attempt, we investigated the Casimir energy on a supersymmetry-breaking brane as dark energy based on the ADD-like geometry [6]7. But instead of invoking Eq.(1) as our guidance, we looked for the general constraint on the various fundamental energy scales if the Casimir energy so induced was interpreted as the dark energy. In Sec.2 we will first correct a mistake in our previous work due to an incorrect normalization convention. We will show, under the corrected expression, that the ADD-required extra-dimension size would provide the right scaling for Casimir energy as dark energy if and only if the number of extra dimension $n=2$. We will comment on the interesting implication of this conclusion.

In Section 3 we will apply the same guiding principle to the RS warped geometry. We invoke the very interesting work by Gherghetta and Pomarol (PG) [8, where SUSY-breaking in the RS model is induced not by dynamics, but by a clever choice of different boundary conditions between the bosonic and the fermionic bulk fields. There the SUSY-breaking induced zero-mode gravitino mass is precisely doubly suppressed from the Planck scale. Unfortunately the higher modes in the graviton-gravitino Kalaza-Klein tower in this case are at the $\mathrm{TeV}$ scale. These higher modes would bring the resultant Casimir energy back to the $\mathrm{TeV}$ scale, and therefore their contributions would overwhelm that from the zero-mode. Thus the PG construction is unfortunately not compatible with our approach to the dark energy problem. Comments and remarks on possible remedies are given at the end.

\section{CASIMIR ENERGY IN ADD GEOM- ETRY}

Casimir effect has been considered as a possible origin for the dark energy by many authors 910111213677. It is known that the conventional Casimir energy in the ordinary $3+1$ dimensional spacetime cannot provide repulsive gravity necessary for dark energy. Conversely, Casimir energy on a 3-brane imbedded in a higher-dimensional world with suitable boundary conditions can in principle give rise to a positive cosmological constant. Typically, the resulting Casimir energy density on the 3-brane scales as

$\rho_{\text {Casimir }}^{(4)} \sim a^{-4}$,

where $a$ is the extra dimension size. As summarized by Milton [9], the required extra dimension sizes for it to conform with the supposed dark energy would have to be very large, roughly consistent with that required for the ADD solution to the Planck-SM hierarchy.

The scaling of the Casimir energy is modified if the system is supersymmetric in the bulk but broken on the brane. In addition to the extra di- 
mension size, the mass-shift of the bulk field under SUSY-breaking also plays a role. Consider a $(3+n+1)$-dimensional space-time with $n$ compact extra dimensions of size $a$, in which the standard model fields and their superpartners are localized on a 3-brane while the gravity sector resides in the bulk. We assume that SUSY is preserved in the bulk and only broken on the 3-brane with a breaking scale $M_{\text {SUSY }}$. In this scenario and at tree level the graviton remains massless everywhere while the gravitino acquires a mass, $m_{3 / 2}$, only on the brane. That is, the gravitino mass is a delta-function along the extra dimension and localized on the brane.

For the ADD model the compactified $n$ extra dimensions can be treated as an $n$-torus, $\mathcal{T}^{n}$. In $\mathcal{M}^{4} \times \mathcal{T}^{n}$ the renormalized Casimir energy density $\rho_{\mathrm{V}}^{(\text {ren })}\left(m^{2}, a\right)$ is the difference of the vacuum energy densities at $a$ and infinity:

$\rho_{\mathrm{V}}^{(\text {ren })}\left(m^{2}, a\right)=\rho_{\mathrm{V}}\left(m^{2}, a\right)-\rho_{\mathrm{V}}\left(m^{2}, a \rightarrow \infty\right)$.

In our SUSY setup and up to $\mathcal{O}\left(m^{2} \phi^{2}\right)$, the $4 \mathrm{D}$ Casimir energy density is the sum of that contributed from graviton and gravitino modes, with the extra dimensions integrated out $[\underline{6}$. This gives

$$
\begin{aligned}
\rho_{\text {Casimir }}^{(4)} & \equiv \rho_{\text {gravitino }}^{(\text {ren })}\left(m_{3 / 2}^{2}, a\right)+\rho_{\text {graviton }}^{(\text {ren })}(0, a) \\
& \cong C_{n} \cdot a^{-2} \cdot m_{3 / 2}^{2} .
\end{aligned}
$$

The coefficient $C_{n}$ is equal in magnitude but opposite in signs for graviton and gravitino modes, and is in general insensitive to the extra dimensionality $n$.

In our previous work the normalization of the $(4+n)$ D mass function was incorrectly treated. As a result, the Casimir energy on the brane had an extra suppression factor $(\delta / a)^{n}$, where $\delta$ is the "thickness" of the brane where SUSY is broken, which we assumed to be the string length. As a consequence $(\delta / a)^{n}$ provided a huge suppression of the Casimir energy. We now recognize that this suppression factor was spurious. If the mass function was properly normalized, this suppression factor should have disappeared.

As we are motivated to connect the two hierarchies, we invoke the ADD relation for the large extra dimension size where the effective gravity strength on the brane is identified with the SM, or $\mathrm{TeV}$, scale:

$a \sim M_{\mathrm{Pl}}^{2 / n} M_{\mathrm{SM}}^{-(n+2) / n}$.

On the ground of dimensional arguments, the gravitino mass is related to the SUSY-breaking scale as

$m_{3 / 2} \sim \frac{M_{\mathrm{SUSY}}^{2}}{M_{\mathrm{Pl}}}$.

Inserting these into Eq.(5), we find

$\rho_{\text {Casimir }}^{(4)} \sim\left(\frac{M_{\mathrm{SUSY}}}{M_{\mathrm{Pl}}}\right)^{4}\left(\frac{M_{\mathrm{SM}}}{M_{\mathrm{Pl}}}\right)^{4 / n-2} M_{\mathrm{SM}}^{4}$.

On the other hand, Eq.(1) gives

$\rho_{\mathrm{DE}} \sim\left(\frac{M_{\mathrm{SM}}}{M_{\mathrm{Pl}}}\right)^{4} M_{\mathrm{SM}}^{4}$.

Following the spirit of Eq.(1), where there are only three energy scales in the world, we assume that the SUSY-breaking scale is also around $\mathrm{TeV}$ : $M_{\mathrm{SUSY}} \sim M_{\mathrm{SM}} \sim \mathrm{TeV}$. If we identify the Casimir energy as the dark energy, i.e., that we equate Eq.(8) and Eq.(9), then our notion would true only if the number of extra dimension $n=2$. This is remarkable and some comments are in order.

We note that there is a fundamental difference between such a Casimir energy and the conventional vacuum energy. The Casimir energy is nontrivial only around the 3 -brane, and it entails the equations of state: $p_{a}=-\rho$ and $p_{b}>0$, where $p_{a}$ and $p_{b}$ are its pressures along the 3 -brane and the extra dimensions, respectively. In contrast, the brane tension from the conventional vacuum energy obeys the following equations of state instead: $p_{a}=-\rho$ and $p_{b}=0$. Study shows that the brane tension can be perfectly cancelled with the curvature via the self-tuning mechanism if the number of extra dimensions is precisely two 14. On the other hand the Casimir energy cannot be removed by the same self-tuning procedure and should survive as the leading contribution to vacuum energy on the brane. We see that our conclusion of $n=2$ coincides with that required by the self-tuning mechanism. Therefore $n=2$ is not only a necessary but also a sufficient condition in 
order for the Casimir energy to behave as dark energy, both qualitatively and quantitatively.

It is interesting to note that a similar concept of SUSY-breaking in a brane-world, called supersymmetric large extra dimensions (SLED), has been invoked to address the vacuum energy and the cosmological constant problem [12]13. In that proposal SUSY in the bulk is broken by the presence of non-supersymmetric 3-branes imbedded in two extra dimensions. Here we arrive at the same conclusion on the $n=2$ extra dimensionality, but through a different route. We note that the same conclusion was reached by Gupta 10 through the study of the KK modes contribution to the vacuum energy in the ADD geometry.

\section{CASIMIR ENERGY IN RS GEOME- TRY}

We now turn our attention to the RS model. Randall and Sundrum introduce the following metric in a $5 \mathrm{D}$ brane world,

$d s^{2}=e^{-2 k a|y|} \eta_{\mu \nu} d x^{\mu} d x^{\nu}+a^{2} d y^{2}$,

where $\mu, \nu=0,1,2,3,-\pi<y<\pi$, and $a$ is the radius of the orbitfold $\mathcal{S}^{1} / \mathcal{Z}_{2}$ in the 5 th dimension $y$. The hidden, or Planck, brane locates at $y=0$ while the visible, or $\mathrm{TeV}$, brane locates at $y=$ $\pi$. As is well-known, the Planck-SM hierarchy is bridged if $k a \sim \mathcal{O}(10)$ so that the mass scale at $y=\pi$ is suppressed by the warp factor,

$\frac{M_{\mathrm{SM}}}{M_{\mathrm{Pl}}} \sim e^{-\pi k a} \sim 10^{-16}$.

It is customary to take $k \sim M_{\mathrm{Pl}}$. So in the RS model the extra dimension size $a$ is only about 10 times the Planck length. We follow the original RS construct where only the gravity sector lives in the bulk while all other fields in the standard model are confined on the $\mathrm{TeV}$ brane. For our purpose we impose supersymmetry to the system. Supersymmetric RS model has been investigated by various authors 15]16. Since we are concentrating on the SUSY-breaking of the gravity sector, it suffices the purpose to consider only the graviton and gravitino fields in the action [8]:

$S=S_{5}+S_{y=0}+S_{y=\pi}$, where the action in the bulk is,

$$
\begin{aligned}
S_{5} & =\int d^{4} x \int d y \sqrt{-g}\left[-\frac{1}{2} M_{5}^{3}(\mathcal{R}\right. \\
& \left.+i \bar{\Psi}_{M}^{i} \gamma^{M N P} D_{N} \Psi_{P}^{i}-i \frac{3}{2} \sigma^{\prime} \bar{\Psi}_{M}^{i} \gamma^{M N} \sigma_{3}^{i j} \Psi_{N}^{j}\right) \\
& \left.-\Lambda_{5}\right],
\end{aligned}
$$

and that on the two branes are

$S_{y=y^{*}}=\int d^{4} x \sqrt{-g}\left[\mathcal{L}_{y^{*}}-\Lambda_{4, y^{*}}\right]$.

Here $\mathcal{R}$ is the $5 \mathrm{D}$ Ricci scalar and $\bar{\Psi}^{i}$ the $5 \mathrm{D}$ symplectic Majorana gravitino fields $(i=1,2)$ whose left-handed and right-handed components satisfy the condition $\gamma_{5} \Psi_{L, R}= \pm \Psi_{L, R}$, respectively, and $\Psi_{\mu L}\left(\Psi_{\mu R}\right)$ are even (odd) under $\mathcal{Z}_{2^{-}}$ parity. Supersymmetry automatically ensures the conditions $\Lambda_{5} / k=\Lambda_{4, \pi}=-\Lambda_{4,0}$. From our motivation, we further assume that these are all identically zero. At $y^{*}=0$ the mass scale is of the order Planck scale, $M_{\mathrm{Pl}}^{2} \sim M_{5}^{3} / k$. With $k \sim M_{\mathrm{Pl}}$, we have $M_{5} \sim M_{\mathrm{Pl}} \sim \mathrm{TeV}$. The effective mass scale on the $y^{*}=\pi$ brane is then of the order $M_{\mathrm{Pl}} e^{-\pi k a}$. In the last term of the bulk action, $\sigma^{\prime} \equiv d(k|y|) / d y= \pm k$ for $y \geq 0$ and $y<0$, respectively.

The Kaluza-Klein (KK) decomposition and the associated eigen-modes for bosons and fermions in the RS geometry have been well studied in recent years 18/19/16. Goldberger and Wise 18. first studied the behavior of bulk scalar field in the RS model. Flachi et al. [19] investigated that for the bulk fermion field. Gherghetta-Pomarol (GP) [16] extended the study to supersymmetric bulk fields in the RS AdS geometry.

The bulk gravitino satisfies the 5D RaritaSchwinger equation,

$\gamma^{M N P} D_{N} \Psi_{P}-\frac{3}{2} \sigma^{\prime} \gamma^{M P} \Psi_{P}=0$.

Decomposing the $5 \mathrm{D}$ gravitino, $\Psi_{M}$, and the 5D supersymmetric parameter, $\eta$, as

$$
\begin{aligned}
\Psi_{\mu L, R}\left(x^{\mu}, y\right) & =\sum_{n} \psi_{\mu L, R}^{(n)}\left(x^{\mu}\right) f_{L, R}^{(n)}(y), \\
\Psi_{5 L, R}\left(x^{\mu}, y\right) & =\sum_{n} \psi_{5 L, R}^{(n)}\left(x^{\mu}\right) f_{5 L, R}^{(n)}(y), \\
\eta_{L, R}\left(x^{\mu}, y\right) & =\sum_{n} \eta_{L, R}^{(n)}\left(x^{\mu}\right) f_{L, R}^{(n)}(y),
\end{aligned}
$$


GP solved the equation of motion and found the $y$-dependent gravitino wavefunctions as

$f_{L}^{(n)}=\frac{e^{3 k|y| / 2}}{N_{n}}\left[J_{2}\left(\frac{m_{n}}{k e^{-k y}}\right)+b Y_{2}\left(\frac{m_{n}}{k e^{-k y}}\right)\right]$,

$f_{R}^{(n)}=\frac{e^{3 k|y| / 2}}{N_{n}}\left[J_{1}\left(\frac{m_{n}}{k e^{-k y}}\right)+b Y_{1}\left(\frac{m_{n}}{k e^{-k y}}\right)\right]$,

where $m_{n}$ is the $4 \mathrm{D}$ gravitino mass for the $n$th mode and the coefficient $b$ satisfies the condition

$b\left(m_{n}\right)=-\frac{J_{1}\left(m_{n} / k\right)}{Y_{1}\left(m_{n} / k\right)}=b\left(m_{n} e^{\pi k a}\right)$.

Solving this equation, the $4 \mathrm{D}$ KK gravitino masses are found to be

$m_{n} \simeq\left(n+\frac{1}{4}\right) \pi k e^{-\pi k a} \quad(n>0)$.

Note that for the massless zero mode $(n=0)$, the wavefunction is localized at the Planck brane:

$f_{L}^{(0)}(y)=\frac{1}{\sqrt{N_{0}}} e^{-k|y| / 2}$.

The behavior of the graviton modes are similar. In particular Randall and Sundrum [20] have shown that the graviton zero mode is also localized at the Planck brane. The 4D mass spectrum for the graviton is identical to that of the gravitino.

Invoking opposite fermion chiralities on the two boundaries 8 , i.e.,

$$
\begin{aligned}
\psi(0) & =\gamma_{5} \psi(0), \\
\psi(\pi a) & =-\gamma_{5} \psi(\pi a),
\end{aligned}
$$

supersymmetry is thus broken on the $\mathrm{TeV}$ brane. Gherghetta and Pomarol show that this would induce a mass shift for the massless zero mode at the tree level which is doubly suppressed by the warp factor,

$m_{3 / 2} \simeq \sqrt{8} k e^{-2 \pi k a} \sim\left(\frac{M_{\mathrm{SM}}}{M_{\mathrm{Pl}}}\right)^{2} M_{\mathrm{Pl}}$.

Physically, such a double suppression from the Planck scale by the RS warp factor is due to the fact that the zero mode wavefunction is localized on the Planck brane, which results in an extra warp factor suppression from the $\mathrm{TeV}$ scale. On the other hand the graviton, which satisfies the periodic, or untwisted, boundary condition, whose zero mode would remain massless at the tree level. Thus the zero mode gravitino-graviton mass split is $\Delta m_{0}=m_{3 / 2}$.

We are now ready to look for the Casimir energy under SUSY-breaking. Casimir energy in the RS geometry has been investigated by several authors 2122 23. First we examine the form of the 4D Casimir energy density under the RS geometry. This can be most easily obtained by introducing a new variable $z=e^{k a|y|} / k$ and rewrite the RS metric in a form which is conformally flat in 5D:

$d s^{2}=\frac{1}{k^{2} z^{2}} \eta_{M N} d x^{M} d x^{N}$,

where $M, N=0, \ldots, 4$. In these conformal coordinates the exponential warp factor disappears, but the separation between the Planck and TeV branes become far apart, i.e., $z_{\pi}-z_{0}=k\left(e^{\pi k a}-\right.$ 1) 17.

Based on these conformal coordinates, one can readily transcribe from what deduced in Sec. 2 the generic scaling of the Casimir energy in the RS model,

$\rho_{\text {Casimir }}^{(4)} \sim \mp e^{-4 \pi k a} a^{-4}$,

for graviton and gravitino fields, respectively. Thus the net Casimir energy is identically zero if SUSY is preserved. Under SUSY-breaking, the scaling becomes

$\rho_{\text {Casimir }}^{(4)} \sim e^{-4 \pi k a} a^{-2} \Delta m^{2}$.

Identifying $k \sim 10 / a \sim M_{\mathrm{Pl}}$ and ignore the numerical factors, the zero-mode contribution to the Casimir energy density conforms with what our inverted hierarchy Ansatz implies:

$\rho_{\text {Casimir }, 0}^{(4)} \sim\left[\left(\frac{M_{\mathrm{SM}}}{M_{\mathrm{Pl}}}\right)^{2} M_{\mathrm{Pl}}\right]^{4} \sim \rho_{\mathrm{DE}}$.

Unfortunately, unlike the zero mode the higher KK mode wavefunctions are localized on the TeV brane instead. As such the mass of the $n$th KK mode for gravitino under twisted boundary condition is shifted to (for $m_{n} \ll k$ and $k a \gg 1$ )

$m_{n} \sim\left(n+\frac{3}{4}\right) \pi k e^{-\pi k a}$, 
while that for the corresponding graviton KK mode under the untwisted boundary condition remains unchanged. Therefore the mass-split between graviton and gravitino for the $n$th mode is of the order

$\Delta m_{n} \sim \frac{\pi}{2} k e^{-\pi k a} \sim M_{\mathrm{SM}} \sim \mathrm{TeV}$.

This energy scale certainly overshadows that of the zero-mode, and our hard-earned minute Casimir energy would be totally overwhelmed.

\section{SUMMARY}

Recent observational evidence indicates that the dark energy may actually be the cosmological constant. We argue that the numerical coincidence between the SM-Planck hierarchy and the inverted SM-DE hierarchy implies a deeper connection between the two. Invoking this connection as our guidance, we investigate the possibility of interpreting the Casimir energy density induced in a SUSY-breaking brane world as the dark energy. We found that for ADD geometry this scenario works if and only if the extra dimensionality is $n=2$. For the RS geometry, we found that based on the GP SUSY-breaking boundary condition the graviton-gravitino KK zero mode contributes to a Casimir energy which conforms with the inverted hierarchy structure. Unfortunately the higher KK modes under the same construction would develop a mass shift at $\sim \mathrm{TeV}$ scale, which overshadows the zero mode contribution. Our attempt is therefore unsuccessful.

One possible way to ameliorate this difficulty is to device a different mechanism for SUSYbreaking under which the higher KK modes would acquire mass shifts that is at or above the Planck scale and would thus be cut off. Conversely, if there is a way where the higher KK mode mass shifts can also be suppressed to the same level as that of the zero mode, then the total Casimir energy contributed from all modes might still retain the same energy scale. These possibilities will be further investigated.

\section{REFERENCES}

1. D. N. Spergel at al., astro-ph/0603449 (2006).
2. M. Tegmark et al., astro-ph/0608632 (2006).

3. P. Astier at al., A\&A 447, 31 (2006).

4. N. Arkani-Hamed, S. Dimopoulos and G. Dvali, Phys. Lett. B 429, 263 (1998).

5. L. Randall and R. Sundrum, Phys. Rev. Lett. 83, 3370 (1999).

6. P. Chen and Je-An Gu, arXiv:astro-ph/0409238 (2004).

7. P. Chen and Je-An Gu, eConf C041213, 1110 (2004).

8. T. Gherghetta and A. Pomarol, Nucl. Phys. B 602, 3 (2001) arXiv:hep-ph/0012378.

9. K. A. Milton, Grav. Cosmol. 9, 66 (2003).

10. A. Gupta, arXiv:hep-th/0210069 (2002).

11. F. Bauer and M. Lindner, arXiv:hep-ph/0309200 (2003).

12. Y. Aghababaie, C. P. Burgess, S. L. Parameswaran and F. Quevedo, Nucl. Phys. B 680, 389 (2004) arXiv:hep-th/0304256.

13. C. P. Burgess, Annals Phys. 313, 283 (2004) arXiv:hep-th/0402200.

14. J. W. Chen, M. A. Luty and E. Ponton, JHEP 0009, 012 (2000).

15. R. Altendorfer, J. Bagger and D. Nemeschansky, Phys. Rev. D63, 125025 (2001) arXiv:hep-th/0003117.

16. T. Gherghatta and A. Pomarol, Nucl. Phys. B 286, 141 (2000) arXiv:hep-ph/0003129.

17. R. Rattazzi, "Cargese Lectures on Extra Dimensions", CERN-PH-TH/2006-029 arXiv:hep-ph/0607055.

18. W. D. Goldberger and M. B. Wise, Phys. Rev. D60, 107505 (1999) arXiv:hep-ph/9907218.

19. A. Flachi, I. G. Moss and D. J. Toms, Phys. Rev. D64, 105029 (2001) arXiv:hep-th/0106076.

20. L. Randall and R. Sundrum, Phys. Rev. Lett. 83, 4690 (1999).

21. W. Naylor and M. Sasaki, Phys. Lett. B 542, 289 (2002).

22. M. R. Satare, arXiv:hep-th/0308109 (2003).

23. A. A. Saharian, Nucl. Phys. B 712, 196 (2003) arXiv:hep-th/0312092. 